\title{
SEARCHING FOR ORBITS AROUND EUROPA THAT REQUIRES LOWER FUEL CONSUMPTION FOR STATIONKEEPING
}

\author{
Jean Paulo dos Santos Carvalho* Rodolpho Vilhena de Moraes* Antônio F. Bertachini \\ de Almeida Prado ${ }^{\dagger}$ Josué Cardoso dos Santos ${ }^{\ddagger}$ \\ * UNIFESP- Instituto de Ciência e Tecnologia, Universidade Federal de São Paulo \\ São José dos Campos - SP, Brazil \\ ${ }^{\dagger}$ Division of Space Mechanics and Control - INPE \\ São José dos Campos - SP, Brazil \\ †UNESP-Faculdade de Engenharia de Guaratinguetá \\ Guaratinguetá-SP, Brazil
}

Email: jeanfeg@gmail.com, rodolpho.vilhena@gmail.com, prado@dem.inpe.br, josuesantosunesp@gmail.com

\begin{abstract}
Nowadays, there are several studies for missions that will place a satellite around Europa. There are many important aspects that deserve to be studied in this natural satellite of Jupiter. It makes the study of orbits around Europa a particular important part of the mission, since a good choice for them will reduce the costs related to station-keeping and then increasing the duration of the mission. In some previous studies, a search for frozen orbit around Europa was presented based in average techniques. The present research has the objective of using the new concept of stability of orbits with respect to station-keeping maneuvers that is available in the literature to study circular orbits around Europa. This concept is based in the integral of the perturbing forces over the time. This value can estimate the total variation of velocity required by the station-keeping propulsion system to compensate the perturbations suffered by the spacecraft. The value of this integral is a characteristic of the perturbations considered and the orbit chosen for the spacecraft. Numerical simulations are made showing the costs of station-keeping for circular orbits around Europa are shown as a function of the eccentricity and semi-major axis of the orbits.
\end{abstract}

Keywords- Astrodynamics, Stability of orbits, Artificial satellites, Planetary satellite, Station-keeping maneuvers.

Resumo- Hoje em dia, existem vários estudos para missões que vão colocar um satélite em torno de Europa. Há muitos aspectos importantes que merecem ser estudados neste satélite natural de Júpiter. Isso torna o estudo das órbitas em torno Europa uma parte importante da missão, uma vez que uma boa escolha para estas órbitas vai reduzir os custos relacionados com estação orbital de manutenção e, em seguida, aumentar a duração da missão. Em alguns estudos anteriores, uma busca por órbita congelada em torno Europa foi apresentada com base em técnicas de média. A presente pesquisa tem o objetivo de utilizar o novo conceito de estabilidade de órbitas com relação às manobras que estão disponíveis na literatura para estudar órbitas circulares em torno de Europa. Este conceito é baseado na integral das forças perturbadoras ao longo do tempo. Este valor pode estimar a variação total da velocidade exigida pelo sistema de propulsão da estação orbital de manutenção para compensar as perturbações sofridas pela sonda. As simulações numéricas são feitas mostrando os custos com manutenção orbital para órbitas circulares em torno Europa com respeito ao semi-eixo maior e a excentricidade destas órbitas.

Palavras-chave- Astrodinâmica, estabilidade, satélites artificiais, satélite planetário, manobras.

\section{INTRODUCTION}

The problem of transfer maneuvers for spacecrafts is very important in space activities. There are many aspects to be considered, like the duration of the transfer, the fuel consumed, etc. The first important result was obtained by Hohmann, (1925), that found the solution for the transfer between two circular and coplanar orbits by applying the minimum variation of velocity to the spacecraft. Later, this type of maneuver that uses impulses as the control available was studied again by several researches. Some of them are Hoelker and Silber (1959), Jin and Melton (1991), Bender (1962), Eckel (1982), Gross and Prussing (1974), Prussing and Chiu (1986), Prado and Broucke (1995, 1996), Prado (1996) and Santos et al. (2012). Studies related to orbits of spacecrafts around Europa can be seen in Scheeres et al. (2001), Lara and Russell (2006), Paskowitz and Scheeres (2006), Lara (2008), Paskowitz and Scheeres (2009), Russell and Brinckerhoff (2009), Lara (2010) and Carvalho et al. (2012a, 2012b).

The goal of the present paper is to perform the integral over the time of all the perturbations for circular orbits around Europa, in order to evaluate the fuel consumption required to keep this orbit keplerian, so compensating the perturbations suffered by the spacecraft. This idea is explained in Prado (2013) and assumes that it is possible to use an ideal propulsion system to deliver a force that has the same magnitude, but in the opposite direction of the perturbations acting in the satellite. It is a kind of stability concept based in the total effects along the time that the perturbations cause in the motion of the spacecraft. So, this value 
Table 1: Numerical values for $J_{2}, J_{3}$ and $C_{22}$

Harmonics coefficients for the Europa

(Lara and Russel, 2006)

$J_{2}=4.355 \times 10^{-4}$

$J_{3}=1.3784 \times 10^{-4}$

$C_{22}=1.3065 \times 10^{-4}$

represents the total variation of velocity that the propulsion system needs to deliver. Applications studying the effects of the third-body perturbation of the Sun and the Moon are also shown in Prado (2013). To make a first study for orbits around Europa using this concept, only circular orbits will be considered, but with semi-major axis and inclinations free to assume any desired value. In this way, the results will allow mission designers to choose the best orbits that are suitable for their missions.

This new criterion of stability has several advantages related to the study of stationkeeping problems like the one shown here. Some of them are:

1) The orbits are always keplerian, so this index can be evaluated for each perturbation independent of the others. In this way, it is possible to make a comparison of those values to chose which forces are important and needs to be considered in the dynamical model, depending on the accuracy desired by the mission;

2) This index depends on the force model used and on the orbit of the spacecraft;

3) It measures the total amount of variation of the velocity that comes from the perturbation forces considered in the dynamical model, so it also represents the consumption of fuel needed to maintain a keplerian orbit for the spacecraft. In this way, this number shows which orbits requires more fuel consumption for the stationkeeping maneuvers;

4) This index depends on the initial position of the spacecraft. So, in order to have a more accurate view, it is necessary to make an average over a certain number of orbits and not taken the result from only one orbit.

\section{MATHEMATICAL MODELS}

The mathematical model used here is now explained in detail. It is assumed that there is a spacecraft in a circular orbit around Europa. The forces acting in this spacecraft is the gravitational field of Europa, with the terms $J_{2}, J_{3}$ and $C_{22}$, and the third-body perturbation due to Jupiter. Table 1 shows the numerical values of those constants. Note that the values are of the same order of magnitude, so it is necessary to take all of them
Table 2: Physical parameters

\begin{tabular}{ll} 
Jupiter & Europa \\
\hline$N=2.0477 \times 10^{-5} \mathrm{rad} / \mathrm{s}$ & $\mu_{E}=3202.7 \mathrm{~km}^{3} / \mathrm{s}^{2}$ \\
\hline
\end{tabular}

into the model. The equations that describe the force field are also shown below.

\subsection{FUNCTION FORCE DUE TO THE DIS- TURBING BODY}

For the model considered in the present paper, it is necessary to calculate the terms $R_{2}$ of the disturbing function due to perturbation caused by a third-body in circular orbit (Jupiter is considered). The disturbing potential $R_{2}$ can be written in the form (Carvalho et al., 2010)

$$
R_{2}=\frac{1}{2} N^{2} r^{2}\left(3 \cos ^{2}(S)-1\right)
$$

where $\mathbf{r}$ is the radius vector of the artificial satellite and $N$ is mean motion of Jupiter. Here, $S$ is the angle between the line that connects the massive central body and the perturbed body (the artificial satellite) and the line that connects the massive central body and the perturbing body (the third body). The artificial satellite is considered as a point mass particle in a circular orbit with osculating orbital elements: $a=r$ (semimajor axis), $i$ (inclination), $g$ (argument of the periapsis), $h$ (longitude of the ascending node). The numerical values for the physical parameters of Jupiter and Europa are shown in Table 2.

Using the relation between the angle $S$ and the true anomaly $(f)$ of the satellite we get (Broucke, 2003)

$$
\cos S=\alpha \cos f+\beta \sin f
$$

For the case of circular orbits, the products $\alpha$ and $\beta$ can be written in the form (Broucke, 2003; Prado, 2003)

$$
\begin{aligned}
& \alpha=\cos g \cos (h-M)-\cos i \sin g \sin (h-M), \\
& \beta=-\sin g \cos (h-M)-\cos i \cos g \sin (h-M) .
\end{aligned}
$$

where $M$ is the true anomaly of Jupiter. For the case of elliptical orbits alpha and beta are presented in Domingos et al. (2008) considering the planetary satellite in the same plane of the disturbing body, however when it is taken into account the inclination of the orbit of the disturbing body (in an elliptical orbit) alpha and beta are given in Liu et al. (2012). Now Eqs. (2) and (3) are replaced in Eq. 1. The disturbing potential 
can be written as

$$
\begin{aligned}
R_{2}= & -\frac{3}{8} r^{2} N^{2}\left[\frac{1}{3}-c^{2}-\right. \\
& \frac{1}{2}(c-1)^{2} \cos (2 g-2 h+2 M+2 f)- \\
& \frac{1}{2}(c+1)^{2} \cos (2 g+2 h-2 M+2 f)- \\
& \cos (2 h-2 M)-\cos (2 g+2 f)+ \\
& \left.c^{2} \cos (2 g+2 f)+c^{2} \cos (2 h-2 M)\right]
\end{aligned}
$$

we will use the shortcut $s=\sin i$ and $c=\cos i$.

The potential at distance $r$ from a mass and the force per unit mass there are related by (McCuskey, 1963)

$$
F=-\frac{\partial U}{\partial r}
$$

where $U$ is the potential to be considered.

Replacing the Eq. (4) in Eq. (5) and using $r=a, f=n t, M=N t$, here $n$ is mean motion of the satellite and $t$ is the time, we get

$$
\begin{aligned}
& F R_{2}=\frac{3}{4} a N^{2}\left[\frac{1}{3}-c^{2}-\right. \\
& \quad \frac{1}{2}(c+1)^{2} \cos (2 g+2 h-2 N t+2 n t)- \\
& \quad \frac{1}{2}(c-1)^{2} \cos (2 g-2 h+2 N t+2 n t)- \\
& \cos (2 N t-2 h)+ \\
& c^{2} \cos (2 N t-2 h)-\cos (2 n t+2 g)+ \\
& \left.c^{2} \cos (2 n t+2 g)\right]
\end{aligned}
$$

\subsection{FUNCTION FORCE DUE TO NON- SPHERICAL SHAPE OF EUROPA}

To analyze the motion of a spacecraft around the planetary satellite it is necessary to take into account the Europa nonsphericity. As mentioned above, we only consider the harmonic coefficients $J_{2}, J_{3}$, and $C_{22}$. Recalling that in this work we consider the satellite in a circular orbit around Europa. We get,

a) The zonal perturbation due to the oblateness $\left(J_{2}\right)$ is

$$
\begin{gathered}
F J_{2}=-\frac{3 \epsilon n^{2}}{2 a}\left[1-3 c^{2}-3 \cos (2 n t+2 g)+\right. \\
\left.3 c^{2} \cos (2 n t+2 g)\right]
\end{gathered}
$$

where $\epsilon=J_{2} R_{E}^{2}, R_{E}$ is the equatorial radius of Europa $\left(R_{E}=1560.8 \mathrm{~km}\right)$.

b) The zonal perturbation due to the pearshaped is defined by

$$
\begin{aligned}
& F J_{3}=-\frac{3 \epsilon_{1} n^{2} s}{2 a^{2}}\left[15 s^{2} \sin (n t+g)-\right. \\
& \left.5 s^{2} \sin (3 n t+3 g)-12 \sin (n t+g)\right]
\end{aligned}
$$

where $\epsilon_{1}=J_{3} R_{E}^{3}$.

c) For the sectorial perturbation we get

$$
\begin{aligned}
& F C_{22}=\frac{9 \delta n^{2}}{4 a}\left[(c-1)^{2} \cos (2 n t+2 g-2 h)+\right. \\
& \quad(c+1)^{2} \cos (2 n t+2 g+2 h)+2 \cos (2 h)- \\
& \left.2 c^{2} \cos (2 h)\right]
\end{aligned}
$$

where $\delta=C_{22} R_{E}^{2}$.

\subsection{FORCES INVOLVED IN THE DINAMICS}

The effects of the forces involved in the system given by the Eqs. (6), (7), (8) and (9) are to change the velocity $(V)$ of the satellite according to the physical law:

$$
\int_{0}^{T} F d t=\Delta V
$$

where $F$ is the force by unit of mass, $T$ is the period and $F=F R_{2}+F J_{2}+F J_{3}+F C_{22}$. We call the integral given in Eq. (10) by Perturbation Integral (PI- km/s),

$$
\int_{0}^{T} F d t=P I
$$

The Eq. (11) is numerically integrated to analyze the influence of the perturbations on the artificial satellite orbit around Europa.

\section{RESULTS}

The main goal of the present paper is to show the evolution of the integral of the perturbing forces over the time for a spacecraft around Europa, as explained before.

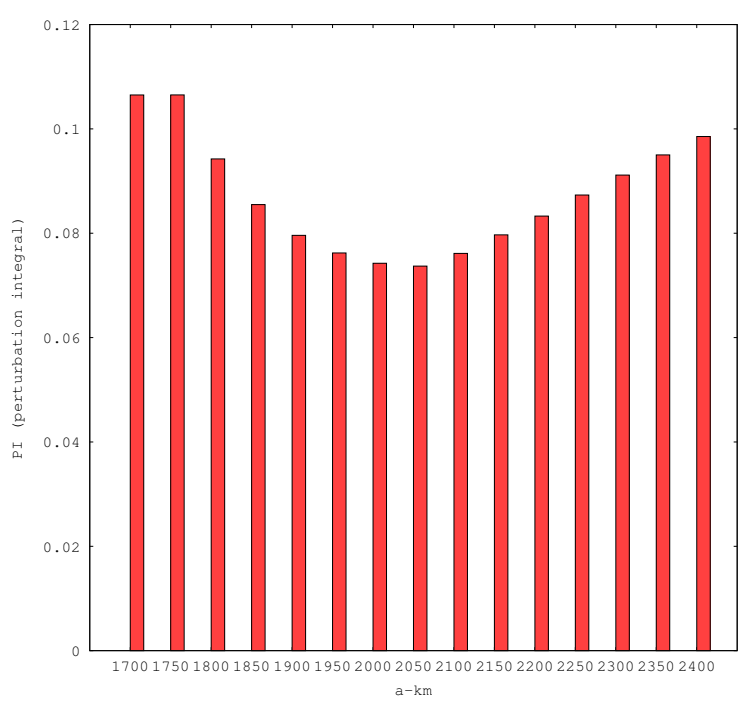

Figure 1: The minimum value for the Perturbing Integral (PI-km/s) as a function of the semi-major axis for polar orbits $\left(i=90^{\circ}\right)$.

Fig. 1 shows the results for the polar orbits, concretizing the semi-major axis and evaluating the Perturbation Integral. It is observed that the 
value of the semi-major axis the gives the minimum value is near $2050 \mathrm{~km}$ for polar orbits. This minimum is about $30 \%$ smaller than the maximum value for the polar case, so a substantial savings in stationkeeping can be obtained by choosing the best altitude, if no other constraint of the mission imposes something different.

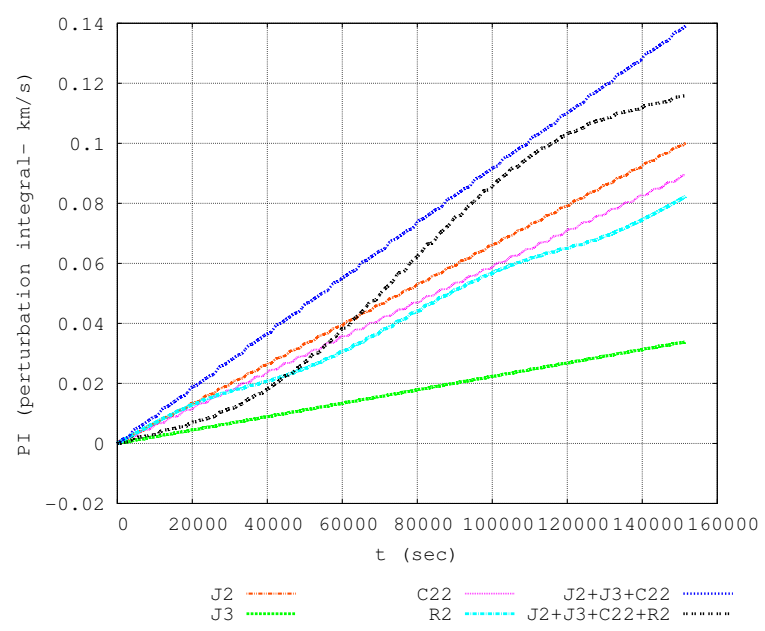

Figure 2: Perturbation Integral (PI) of each force as a function of the time for orbits with semimajor axis of $1670 \mathrm{~km}, g=270^{\circ}, h=90^{\circ}$ and $i=90^{\circ}$.

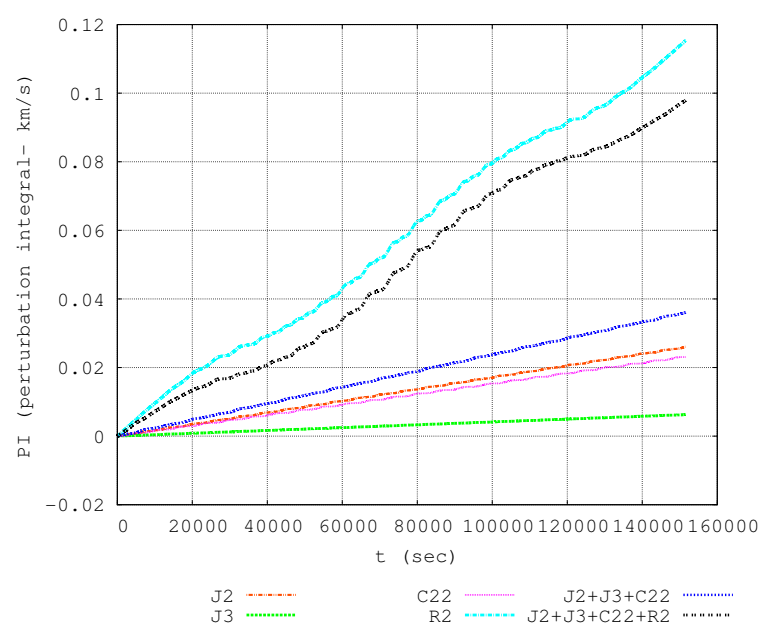

Figure 3: Perturbation Integral (PI) of each force as a function of the time for orbits with semimajor axis of $2341 \mathrm{~km}, g=270^{\circ}, h=90^{\circ}$ and $i=90^{\circ}$.

Fig. 2 and Fig. 3 show the evolution of the magnitude of the integral for all the forces considered in the dynamical model, individually. Studying the results in more detail, it is visible that the sum of the contributions of each individual effect is different from the total effect. This is explained by the fact that the perturbations have positive and negative signs with respect to each other. So, there are compensations, which mean that, for a given position of the spacecraft, some perturbing forces are trying to deviate the orbit from Keplerian, while some other forces are working in the opposite direction, so helping the control system to maintain the orbit keplerian and then reducing the values of the integral and the fuel consumption.

Studying the results in more detail, it is visible that the sum of the contributions of each individual effect is different from the total effect. This is explained by the fact that the perturbations have positive and negative signs with respect to each other. So, there are compensations, which mean that, for a given position of the spacecraft, some perturbing forces are trying to deviate the orbit from Keplerian, while some other forces are working in the opposite direction, so helping the control system to maintain the orbit keplerian and then reducing the values of the integral and the fuel consumption. A clear indication of this fact is shown in Fig. 2, where the Perturbing Integral related to the total forces is smaller than the Perturbing Integral of the effects of the terms only due to the potential of Europa. It clearly means that the third-body perturbation of Jupiter is helping to control the system and reducing the fuel consumption. This is an interesting characteristics of the approach used here to evaluate stability of orbits and emphasize the importance of finding the most important perturbations involved in the dynamics.

The next interesting fact that can be explained is the variation of the Perturbing Integral with respect to the inclination of the orbit of the spacecraft. Fig. 2 shows a polar orbit with semimajor axis of $1670 \mathrm{~km}$. Note that looking at the individual effects of each perturbation, some information can be obtained. The contribution of $J_{3}$ goes from near to 0.038 . Regarding $J_{2}$, its contribution goes from near 0.1 again for the polar orbits. For the $C_{22}$ term, its contribution goes from near to 0.09. As a result of those effects, the total contribution of the potential of Europa goes closes to 0.14 . It is interesting to note that the contribution of the $J_{2}$ term dominates the scene for the polar orbits. It is also necessary to take into account the compensations by having different signs of the forces. Polar orbits have more aligned effects of the individual terms of the potential of Europa. It is also clear that for polar orbits the effects of the total potential are larger than all the individual elements. It should also be noted that, for the polar orbits, the value of the integral for the total perturbations is smaller than the value obtained when considering only the potential of Europa. The third-body perturbation helps the control the system for polar orbits, and this fact makes the costs smaller than the equivalent ones for equatorial orbits.

Looking now to Fig. 3, showing the results 
for orbits with semi-major axis of $2341 \mathrm{~km}$, it is clear that the third-body perturbation of Jupiter starts now to be the main force in the dynamics of the spacecraft. The potential of Europa now plays the role of working against the third-body perturbation, so reducing the value of the integral. But these compensating effects are not the same for all the orbits studied. The larger effects in the polar orbits make the value of the Perturbing Integral to decrease and the minimum of the graph is no longer sharp. Fig. 4 shows that the Perturbing Integral is almost constant from inclination from 70 to 110 degrees. So, this is one more fact to be considered when choosing an orbit for a spacecraft around Europa.

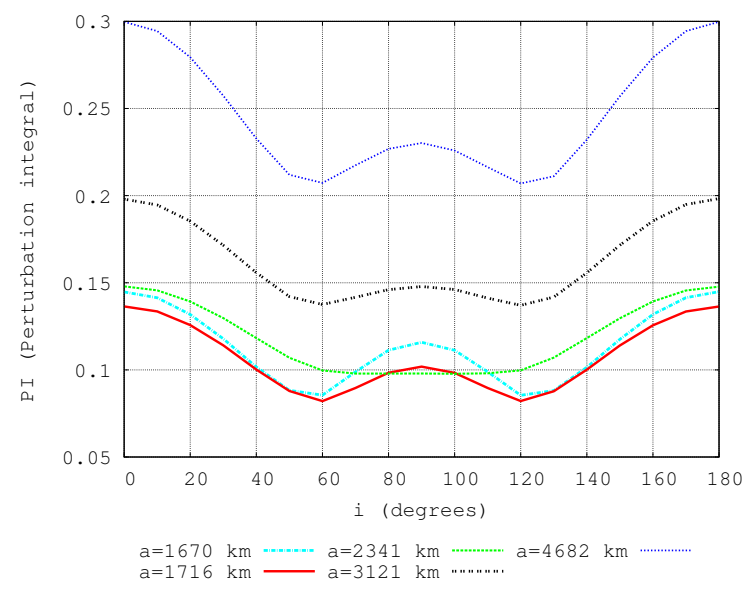

Figure 4: Perturbation Integral (PI-km/s) as a function of the inclination for orbits with semimajor axis of $2341 \mathrm{~km}$

In Carvalho et al. (2013) was found frozen orbits for an artificial satellite around Europa taking into account the non-spherical shape $\left(J_{2}, J_{3}\right)$ of the planetary satellite, and the perturbation of the third body (circular orbit). The orbit found presents the characteristics shown in Fig. 1 where the polar orbit has a lower value for the integral defined herein (PI). With the same approach developed in Carvalho et al. (2013), but now also taking into account the $C_{22}$ term, Fig. 5 was generated to analyze the behavior of polar orbits in the region shown in Fig. 1. Note that we found orbits that librate around the equilibrium point with varying amplitudes. The orbits with eccentricity 0.04 and 0.05 are frozen (Carvalho 2010, 2011), since they have smaller variation in this diagram.

\section{Conclusions}

This paper studied the stability of circular orbits around Europa, using a new proposed index for this comparison, based on the integral of the perturbing forces over the time. This definition showed to be useful in the evaluation of the fuel consumption for station-keeping maneuvers.

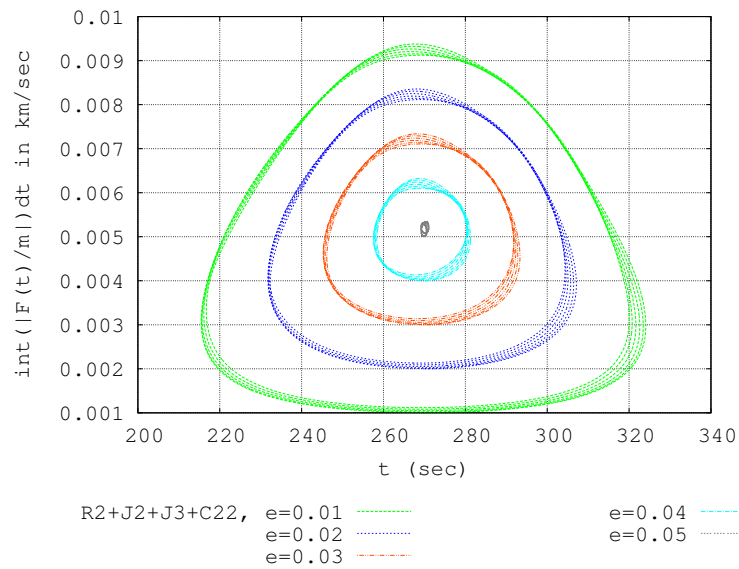

Figure 5: Time evolution (300 days) of the eccentricity $(e)$ and periapsis $(g)$. (a) Initial conditions: $a=2341 \mathrm{~km}, i=90^{\circ}, g=270^{\circ}$ and $h=90^{\circ}$.

A study was then made to know the effects of the main terms of the potential of Europa $\left(J_{2}\right.$, $J_{3}, C_{22}$ ) and the third-body perturbation due to Jupiter. The results showed several interesting characteristics that depends on the orbit of the spacecraft, like: 1) the existence of orbits with minimum value for this index with respect to the semi-major axis, for a fixed inclination; 2) the existence of orbits with minimum value for this index with respect to the inclination, for a fixed semimajor axis; 3 ) the role of each individual term of the perturbing forces; 4) the forces have different signs with respect to each other, so, for a given position of the spacecraft, some forces are acting to destroy the keplerian orbit, while others are working together with the propulsion system to keep the keplerian orbit, so reducing the fuel consumption; 5) orbits with semi-major axis of $2341 \mathrm{~km}$ have a flat minimum, with values for the integral about the same for inclinations ranging from 50 to 90 degrees. With this new approach we find a region with values of semi-major axis, which can be found frozen orbits as shown in Fig 1 and Fig 5. The dynamic model presented here is very simplified, just to test this new concept to analyze the regions where frozen orbits can be found. In another paper we will use the criterion of integral of the force in a more realistic dynamics, considering elliptical orbits.

\section{acknowledgements}

The author are grateful to FAPESP (Foundation to Support Research in São Paulo State) under the contracts N ${ }^{\circ}$ 2011/05671-5 and 2012/21023-6, SPBrazil, CNPq (National Council for Scientific and Technological Development) - Brazil for contracts 304700/2009-6, 3003070/2011-0 and CAPES. 
Bender, D.F., Optimum coplanar two-impulse transfers between elliptic orbits. Aerospace Engineering, pp. 44-52, 1962.

Broucke, R.A., Long-term third-body effects via double averaging. Journal of Guidance, Control, and Dynamics, Vol. 26, no. 1, pp. 27-32, 2003. DOI: $10.2514 / 2.5041$

Carvalho, J. P. S., Vilhena de Moraes, R., Prado A. F. B. A., Dynamics of artificial satellites around Europa. Mathematical Problems in Engineering, Volume 2013, Article ID 182079, 7 pages doi 0.1155/2013/182079, 2013.

DOI: $18207910.1155 / 2013 / 182079$

Carvalho, J.P.S.; Elipe, A.; Moraes, R.V. and Prado, A.F.B.A., Low-altitude, near-polar and near-circular orbits around Europa. Advances in Space Research, Vol. 49, pp. 994-1006, 2012a. DOI: $10.1016 /$ j.asr.2011.11.036

Carvalho, J. P. S.; Mourão, D. C.; Elipe, A.; Moraes, R. V. and Prado, A. F. B. A., Frozen orbits around Europa. International Journal of Bifurcation and Chaos in Applied Sciences and Engineering, vol. 22, no. 10, Article ID 1250240, 13 pages, 2012b.

Carvalho, J. P. S., Vilhena de Moraes, R., Prado A. F. B. A., Planetary Satellite Orbiters: Applications for the Moon, Mathematical Problems in Engineering, Volume 2011, Article ID 187478, 19 pages doi:10.1155/2011/187478, 2011.

Carvalho, J. P. dos S., Vilhena de Moraes, R., Prado, A. F. B. A. Some Orbital Characteristics of Lunar Artificial Satellites, Celest. Mech. Dyn. Astron. Vol. 108 no. 4, pp. 371-388, 2010. DOI: $10.1007 / \mathrm{s} 10569-010-9310-6$

Domingos, R.C.; Moraes, R.V. and Prado, A.F.B.A., Third-body perturbation in the case of elliptic orbits for the disturbing body. Mathematical Problems in Engineering, pp. 1-15, 2008.

DOI: $10.1155 / 2008 / 763654$

Eckel, K.G., Optimal impulsive transfer with time constraint. Astronautica Acta, Vol. 9, no. 3, pp. 139-146, 1982. DOI:10.1016/0094-5765(82)90081-9

Gross, L.R. and Prussing, J.E., Optimal multiple-impulse direct ascent fixed-time rendezvous. AIAA Journal, Vol. 12, no. 7, pp. 885889, 1974. DOI: $10.2514 / 3.49376$

Hoelker, R.F. and Silber, R.. The BiElliptic Transfer Between Circular Co-Planar Orbits. Tech Memo 2-59, Army Ballistic Missile Agency, Redstone Arsenal, Alabama, USA, 1959.

Hohmann, W.. Die Erreichbarkeit der Himmelskorper. Oldenbourg, Munique, 1925.

Jin, H.; Melton, R.G.. Transfers between circular orbits using fixed impulses. AAS paper 91161. In: AAS/AIAA Spaceflight Mechanics Meeting, Houston, TX, 11-13 Feb. 1991.

Lara, M., Russell, R. On the design of a science orbit about Europa, paper 2006 AAS/AIAA, presented at the 2006 Space Flight Mechanics Meeting, Tampa, Florida, January 22-26, 2006.

Lara, M., Simplified equations for computing science orbits around planetary satellites. Journal of Guidance, Control and Dynamics. Vol. 30, no. 1, pp. 172-181, 2008. DOI: 10.2514/1.31107

Lara, M. Three-body dynamics around the smaller primary. application to the design of science orbits, Journal of Aerospace Engineering, Sciences and Applications, Vol. 2, no. 1, 2010.

Liu, X., Baoyin, H., Ma, X., Long-term perturbations due to a disturbing body in elliptic inclined orbit, Astrophysics and Space Science, Vol. 339, no. 2, pp. 295-304, 2012. DOI: $10.1007 / \mathrm{s} 10509-012-1015-8$

McCuskey, S. W. Introduction to Celestial Mechanics, Addison-Wesley Publishing Company, INC., United States of America, 184p., 1963.

Paskowitz, M. P.; Scheeres, D. J. Control of Science Orbits About Planetary Satellites, Journal of Guidance, Control and Dynamics, Vol. 32, no. 1, pp. 223-231, 2009.

Paskowitz, M. E., and Scheeres, D. J., Design of Science Orbits About Planetary Satellites: Application to Europa, Journal of Guidance, Control and Dynamics, Vol. 29, No. 5, pp. 1147-1158, 2006.DOI: $10.2514 / 1.36220$

Prado A.F.B.A. and Broucke, R.A., Transfer orbits in restricted problem. Journal of Guidance Control and Dynamics, Vol. 18, no. 3, pp. 593598, 1995. DOI: 10.2514/1.19464

Prado, A.F.B.A. and Broucke, R.A., Transfer orbits in the Earth-Moon system using a regularized model. Journal of Guidance, Control and Dynamics, Vol. 19, no. 4, pp. 929-933, 1996. DOI: $10.2514 / 3.21428$

Prado, A.F.B.A., Third-body perturbation in orbits around natural satellites. Journal of Guidance, Control and Dynamics, Vol. 26, no. 1, pp. 33-40, 2003. DOI: 10.2514/2.5042

Prado, A.F.B.A., Traveling between the Lagrangian points and the Earth. Acta Astronautica, Vol. 39, no. 7, pp. 483-486, 1996. DOI: $10.1016 /$ S0094-5765(97)85428-8

Prado, A.F.B.A., Searching for Orbits with Minimum Fuel Consumption for Station-Keeping Maneuvers: An Application to Lunisolar Perturbations. Mathematical Problems in Engineering Volume 2013 (2013), Article ID 415015, 11 pages.

Prussing, J.E. and Chiu, J.H., Optimal multiple-impulse time-fixed rendezvous between circular orbits. Journal of Guidance, Control, and Dynamics, 9(1), 17-22, 1986. DOI: 10.2514/3.20060

Russell, R. P., Brinckerhoff, A. T. Circulating eccentric orbits around planetary Moons. Journal of Guidance, Control and Dynamics, Vol. 32, no. 2, pp. 423-435, 2009. DOI: 10.2514/1.38593

Santos, D. P. S., Prado, A. F. B. A., Colasurdo, G., Four-Impulsive Rendezvous Maneuvers for Spacecrafts in Circular Orbits Using Genetic Algorithms. Mathematical Problems in Engineering, Volume 2012, 2012.

Scheeres, D. J., Guman, M. D., Villac, B. F. Stability Analysis of Planetary Satellite Orbiters: Application to the Europa Orbiter. Journal of Guidance, Control and Dynamics, Vol. 24, no. 4, pp. 778-787, 2001. DOI: 10.2514/2.4778 